Eppur si muove: Vor einigen Jahren haben wir auf Initiative der FMH begonnen, mit dem BSV Gespräche zu führen. Grund waren die Veränderungen innerhalb und um die IV, die dazu geführt hatten, dass der behandelnde Arzt im ganzen Prozess noch knapp Informationslieferant war, aber dessen Kompetenzen und Zuständigkeiten von Versicherungen und vor allem auch Gerichten immer mehr in Zweifel gezogen wurden. In einem langen Prozess haben sich Verständnis und Akzeptanz für den anderen etablieren können, und es bleibt die ewige Weisheit: Nur wenn man sich kennt, kann man miteinander einen Weg gehen. Wir sind daran, das Ziel ist klar: respektvolle Zusammenarbeit zum Wohl der Patienten, der Versicherten.

Dr. med. Carlos Beat Quinto,

Mitglied des FMH-Zentralvorstandes, Departementsverantwortlicher Public Health und Gesundheitsberufe Dr. med. Philippe Luchsinger, Präsident Hausärzte Schweiz

\title{
Weiterentwicklung der IV
}

\section{Stefan Ritler}

Vizedirektor Bundesamt für Sozialversicherungen BSV, Leiter Geschäftsfeld IV

Mit der Weiterentwicklung der IV verfolgt der Bundesrat das Ziel, das System IV zu verbessern, unter der Prämisse, die Eingliederung zu verstärken und eine Invalidität zu verhindern. Im Zentrum stehen die intensivere Begleitung und Steuerung bei Geburtsgebrechen, die gezielte Unterstützung von Jugendlichen beim Übergang ins Erwerbsleben und der Ausbau der Beratung und Begleitung von Menschen mit psychischen Gesundheitsstörungen. Zur Erreichung der Ziele will der Bundesrat die Zusammenarbeit zwischen den Ärzten und Arbeitgebenden sowie der IV ausbauen. Die Vorlage sieht zudem vor, das heutige Rentenmodell durch ein stufenloses System zu ersetzen.

Die IV hat sich erfolgreich von einer Renten- zu einer Eingliederungsversicherung gewandelt. Dies wird auch in den Ergebnissen der Evaluationen der IV-Revisionen der letzten zwölf Jahre erkennbar (Beiträge zur Sozialen Sicherheit: Forschungsberichte Nr. 13/12, 2/13 und 18/15). Diese Evaluationen zeigen aber auch, dass bei den Kin-

Um die Zusammenarbeit zu stärken, sollen behandelnde Ärztinnen und Ärzte besser über die IV im Allgemeinen sowie über die Eingliederungsmassnahmen ihrer Patientinnen und Patienten informiert werden.

dern und den Jugendlichen sowie bei Personen mit psychischen Gesundheitsstörungen weitere Massnahmen nötig sind, um die Invalidität zu vermeiden und die Eingliederung zu fördern. Die OECD konnte in ihrer 2014 veröffentlichten Studie zur psychischen Gesundheit und Beschäftigung in der Schweiz aufzeigen, dass es eine Verstärkung der Koordination und Kooperation der IV mit anderen Akteuren des Gesundheits-, des Schul- und des Berufsbildungswesens sowie mit den Arbeitgebern und den Partnerversicherungen braucht, um die Berufschancen von Menschen mit gesundheitlichen Beeinträchtigungen zu erhöhen. Weitere Forschungsarbeiten von Baer et al. 2015 und Bolliger et al. 2015 kamen zum Schluss, dass vor allem die enge $\mathrm{Zu}$ sammenarbeit zwischen den IV-Stellen und den behandelnden Ärztinnen und Ärzten zum Gelingen einer erfolgreichen Eingliederung beitragen kann.

Diese und weitere Erkenntnisse sind in die vorgesehenen Massnahmen für bestimmte Zielgruppen eingeflossen.

\section{Kinder mit Geburtsgebrechen: engere Begleitung und gezieltere Steuerung}

Kindern und Jugendlichen finanziert die IV die medizinische Behandlung von anerkannten Geburtsgebrechen. Künftig will die IV die Kinder und ihre Familien insbesondere bei komplexen gesundheitlichen Einschränkungen enger begleiten. Die medizinischen Behandlungen werden zur Unterstützung der späteren Eingliederung verstärkt mit anderen Leistungen der IV koordiniert. Dazu arbeitet die IV intensiver mit den behandelnden Ärzten zusammen. Gleichzeitig soll die 
Liste der Geburtsgebrechen auf den neusten Stand gebracht werden. Weitere seltene Krankheiten, die den heute bereits bestehenden Kriterien entsprechen, sollen aufgenommen werden. Dagegen sollen Geburtsgebrechen, die heute aufgrund der medizinischen Fortschritte mit geringem Aufwand behandelt werden können, künftig von der Krankenversicherung übernommen werden.

\section{Jugendliche: Übergang ins Erwerbsleben gezielt unterstützen}

Der Bundesrat will keine fixe Altersgrenze festlegen, vor der keine IV-Rente ausbezahlt wird. Dennoch ist es aus seiner Sicht wichtig, dass junge Menschen nicht als Rentner oder Rentnerinnen ins Erwachsenenleben starten. Eine Rente soll daher erst zugesprochen werden, wenn alle Massnahmen zur Eingliederung ausgeschöpft worden sind. Die IV möchte deshalb die Instrumente ausbauen, die Jugendlichen mit psychischen oder anderen Beeinträchtigungen im Übergang von der Volksschule zur ersten beruflichen Ausbildung helfen: Die Beratung und Begleitung von jungen Versicherten wie auch von Fachpersonen aus Schule und Ausbildung soll ausgebaut und verstärkt werden. Die bei Erwachsenen bewährten Instrumente der Früh- erfassung und der sozialberuflichen Integrationsmassnahmen sollen künftig auch Jugendlichen zugutekommen. Die IV soll zudem vorgelagerte kantonale Angebote zur Eingliederung Jugendlicher, insbesondere zur Vorbereitung auf die erste Berufsausbildung und das kantonale Case-Management Berufsbildung, mitfinanzieren können. Die erstmaligen beruflichen Ausbildungen sollen wenn immer möglich im ersten Arbeitsmarkt stattfinden. Neu sollen die Lernenden statt eines - manchmal zu hohen - Taggelds der IV einen Lohn von den Arbeitgebenden erhalten, der jenem von anderen Lernenden entspricht. Junge Menschen in der beruflichen Eingliederung haben zudem fünf Jahre länger als bisher, bis zum 25. Altersjahr, Anspruch auf medizinische Massnahmen der IV.

\section{Psychisch Beeinträchtigte: Beratung und Begleitung ausbauen}

Menschen mit psychischen Beeinträchtigungen brauchen spezifische Unterstützung, damit sie im Arbeitsleben verbleiben oder Eingliederungsmassnahmen erfolgreich abschliessen können. So soll eine Früherfassung künftig noch früher, also bereits bevor es zu einer Krankschreibung kommt, möglich sein. Betroffene Personen sollen zudem frühzeitig und über

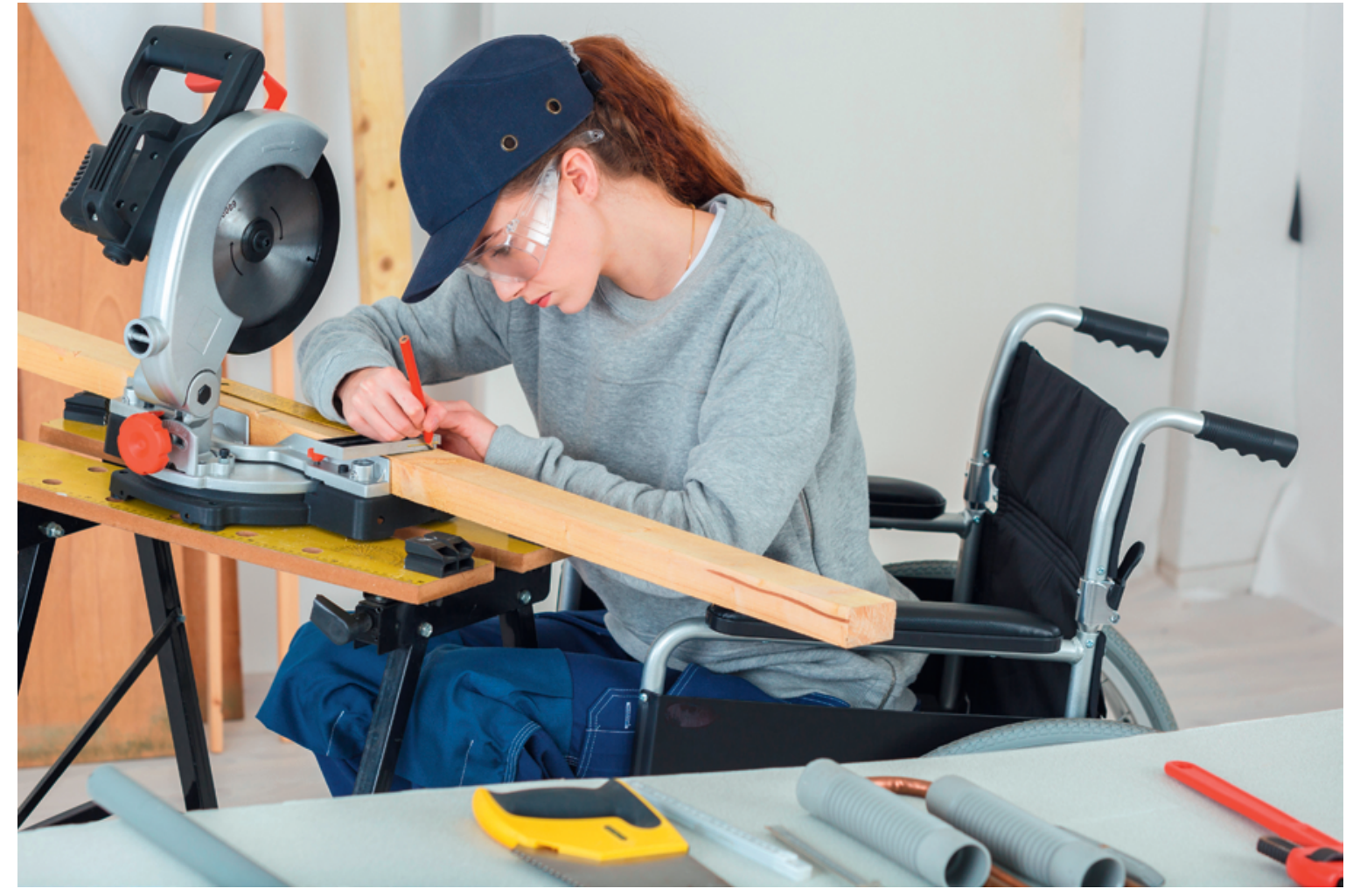

Junge Menschen sollen nicht als Renter oder Rentnerinnen ins Erwachsenenleben starten. Die IV möchte aus diesem Grund Massnahmen ausbauen, die beeinträchtigten Jugendlichen im Übergang von der Volsschule zur ersten beruflichen Ausbildung helfen (Symbolbild). 
die Eingliederung hinaus von der IV begleitet und beraten werden können. Neu wird ein Personalverleih vorgeschlagen, mittels dessen Arbeitgebende potentielle Angestellte kennenlernen können. Die sozialberuflichen Integrationsmassnahmen sollen zeitlich ausgedehnt und besser an individuelle Bedürfnisse angepasst werden. Um die Vermittlungschancen nach Wegfall der Invalidenrente zu erhöhen, soll schliesslich die mögliche Bezugsdauer für Taggelder der Arbeitslosenversicherung auf 180 Tage verdoppelt werden.

\section{IV arbeitet verstärkt mit Ärzten und Arbeitgebenden zusammen}

Die behandelnden Ärztinnen und Ärzte verfügen über eine umfassende Kenntnis der Krankheitsgeschichte und der aktuellen gesundheitlichen Situation einer Person. Sie stellen die Diagnose und können Aussagen zu Schweregrad, Auswirkungen und Prognose eines gesundheitlichen Ereignisses machen. Ihre Behandlungen sind tendenziell eher auf die Behandlung der Symptome und Defizite fokussiert. Für die IV-Stellen steht hingegen die Frage, wie sich eine für den Versicherten zumutbare medizinische Behandlung auf die Eingliederung ins Erwerbsleben auswirkt, im Vordergrund. Da diese unterschiedlichen Rollen nicht grundsätzlich verändert werden können, muss eine Zusammenarbeitskultur etabliert werden, die eine konstruktive und für die betroffene Person wirkungsvolle Fallführung erlaubt.

Um die Zusammenarbeit zu stärken, sollen behandelnde Ärztinnen und Ärzte besser über die IV im Allgemeinen sowie über die Eingliederungsmassnahmen ihrer Patientinnen und Patienten informiert werden. Zudem soll in der ärztlichen Aus-, Fort- und Weiterbildung mehr Platz für versicherungsmedizinische Inhalte eingeräumt werden. Es geht dabei nicht nur um die Stärkung des Vertrauens, vielmehr ist eine verbesserte Information auch eine wichtige Voraussetzung für die Arbeit mit dem Patienten / der Patientin. Zur Erleichterung des gegenseitigen Austauschs wird mit der Weiterentwicklung der IV eine gesetzliche Grundlage geschaffen (Art. 66a Abs. 1 Bst. d E-IVG). Diese Bestimmung entbindet die IV-Stelle von ihrer Schweigepflicht nach Artikel 33 ATSG gegenüber der behandelnden Ärzteschaft. Dies ermöglicht einen raschen und informellen gegenseitigen Informationsaustausch und fördert die Zusammenarbeit. Siehe dazu den Artikel «Werkzeugkasten für Ärztinnen und Ärzte» in dieser Ausgabe.
Am 15. Februar 2017 hat der Bundesrat die Botschaft zur Weiterentwicklung der IV ans Parlament überwiesen. Sie ist nachzulesen unter: https://www.admin.ch/opc/de/federal-gazette/2017/2535. pdf. Die parlamentarische Beratung der Vorlage beginnt in den kommenden Monaten.

Die Weiterentwicklung der IV sieht auch praktische Verbesserungen vor, die es den Arbeitgebenden vereinfachen, gesundheitlich beeinträchtigte Personen bei der Eingliederung zu unterstützen. Die Optimierung und Erweiterung der Massnahmen für die genannten Zielgruppen und die engere Koordination mit beteiligten Akteuren ziehen sich wie ein roter Faden durch die Reform. Die IV soll früher, rascher und gezielter eingreifen können. Dabei spielt die Kommunikation zwischen der IV-Stelle und den behandelnden Ärzten eine entscheidende Rolle: Nur wenn die Massnahmen der IV und die ärztlichen Behandlungen von Beginn an miteinander koordiniert werden, können Ausbildungsabbrüche verhindert, Arbeitsplätze erhalten bzw. Personen bestmöglich wieder eingegliedert und verfrühte Berentungen verhindert werden. Dazu ist es auch nötig, so früh wie möglich, bereits wenn die Gefahr einer Invalidisierung erkennbar wird, zu handeln

\section{Literatur}

- Baer, Niklas; Altwicker-Hàmori, Szilvia; Juvalta, Sibylle; Frick, Ulrich; Rüesch, Peter (2015): Profile von jungen Neurentenbeziehenden mit psychischen Krankheiten; [Bern: BSV]. Beiträge zur Sozialen Sicherheit. Forschungsbericht Nr. 19/15: www.bsv.admin.ch $\rightarrow$ Publikationen \& Service $\rightarrow$ Forschung und Evaluation $\rightarrow$ Forschungspublikationen

- Bolliger, Christian; Féraud, Marius (2015): Die Zusammenarbeit zwischen der IV und den behandelnden Ärztinnen und Ärzten: Formen, Instrumente und Einschätzungen der Akteure; [Bern: BSV]. Beiträge zur Sozialen Sicherheit. Forschungsbericht Nr. 5/15: www.bsv.admin.ch $\rightarrow$ Publikationen \& Service $\rightarrow$ Forschung und Evaluation $\rightarrow$ Forschungspublikationen.

- Isik, Serkan; Wälti, Pia: Schulterschluss für eine rasche Wiedereingliederung. Schweiz Ärztezeitung. 2016;97(9):321-3.

- OECD (2014): Psychische Gesundheit und Beschäftigung: Schweiz; [Bern, BSV]. Beiträge zur Sozialen Sicherheit. Forschungsbericht Nr. 12/13.

- Guggisberg, Jürg; Bischof, Severin; Jäggi, Jolanda; Stocker, Désirée: Evaluation der Eingliederung und der eingliederungsorientierten Rentenrevision der Invalidenversicherung (2015; Berichtnummer 18/15).

- Gehrig, Matthias; Guggisberg, Jürg; Graf, Iris: Wohn- und Betreuungssituation von Personen mit Hilflosenentschädigung der IV (2013; Berichtnummer 2/13).

- Bolliger, Christian; Fritschi, Tobias; Salzgeber, Renate; Zürcher, Pascale; Hümbelin, Oliver: Eingliederung vor Rente. Evaluation der Früherfassung, der Frühintervention und der Integrationsmassnahmen in der Invalidenversicherung (2012; Berichtnummer 13/12).

Bildnachweis

(c) Auremar | Dreamstime.com 